\title{
Tradeoffs in the Application of Time-Reversed Acoustics to Tactile Stimulation
}

\author{
Charles Hudin ${ }^{1}$, José Lozada ${ }^{1}$, Michael Wiertlewski ${ }^{1,2}$, and Vincent Hayward ${ }^{2}$
}

1 CEA, LIST, Sensorial and Ambient Interfaces Laboratory, 91191, Gif-sur-Yvette Cedex, France

2 UPMC Univ. Paris 6, ISIR, Institut des Systèmes Intelligents et de Robotique, 75005, Paris, France

$\{$ charles.hudin, jose.lozada\}@cea.fr,

\{michael.wiertlewski, vincent.hayward\}@isir.upmc.fr

\begin{abstract}
The creation of active tactile surfaces through electromechanical actuation is an important problem. We describe here the application of time-reversed acoustics to the creation of deformations localized in time and in space in a stretched membrane that can be touched. We discuss the basic physical and engineering tradeoffs of this approach and describe the results obtained from an experimental mock-up device.
\end{abstract}

Keywords: Surface Haptics, Time-Reversed Acoustics.

\section{Introduction}

The electromechanical stimulation of the fingertip has attracted the interest of researchers since the early works of Gault $[1,2]$. Recently there has been a lot of interest in providing active surfaces that can stimulate the fingertips mechanically, while permitting free exploration.

This question has been approached by vibrating the entire surface being touched, in the normal or tangential directions, as is commonly done is consumer devices. Another approach is to modulate the interaction force between a finger and a surface during sliding. Surface acoustic waves can be employed to this end, but require the use of an thin intermediary sheet between the finger and the surface $[3,4]$. Nonlinear acoustic pumping applied to tactile stimulation was pioneered by Watanabe and Fukui [5]. This approach, which modifies the finger-surface interaction in a controlled manner, and without intermediary, has been recently further developed $[6,7,8,9]$. Electro-vibration, where the interaction force modulation is achieved by electrostatic attraction between the surface and the skin, originally demonstrated by Strong and Troxel [10], remains popular today owing to its implementation simplicity [11], despite its inherent weakness and sensitivity to physiological and environmental factors [12]. Another direction of investigation is the transport of mechanical energy through waves. Acoustic phase arrays can create significant non-contact stimulation from remotized transducers, as shown by Iwamoto et al. [13]. They were also further developed to create specific tactile patterns [14]. 
Here, we describe a new surface actuation mode, which combines the remotization of the actuators with an active tactile surface delivering the stimulation. It is based on the concept of 'computational time reversal' and is able to stimulate to one or several regions, and hence several fingers, independently with a high temporal and spatial resolution, using a membrane as an acoustic propagation medium. We discuss in this article the basic physical and realization tradeoffs and report on a proof-of-concept device that can displace a surface out-of-plane by 200 micro-meters in a $1 \mathrm{~cm}^{2}$ region.

\section{Time Reversal}

\subsection{Principle}

Time-reversal is a computational technique that takes advantage of a basic property of waves which, at first sight, seems to run counter to the principle of irreversibility [15]. For instance, owing to the irreversibility of certain processes, it is not possible to reconstruct the exact configuration of a broken glass from scattered pieces, even if each single atom on each side of the cracks can be brought back its original place. Macroscopic waves, however, obey a fundamental symmetry arising from the wave equation $\partial^{2} u / \partial t^{2}=c^{2} \nabla^{2} u$. The equation is invariant under substitution of $t$ by $-t$, which means that the initial and final conditions can be interchanged. This property holds even with complex, anisotropic, inhomogenous, nonlinear media, where the right-hand-side, involving the Laplacian operator, can be arbitrarily complicated, provided that it has no memory and does not depend on time. If the medium is dissipative, with terms involving $\partial u / \partial t$, the symmetry is broken, but the losses can be corrected for [16].

Computational time-reversal was originally developed to focus ultrasound waves in inhomogeneous, scattering media such as tissues [17]. Since then, it has been applied to surface waves in water, electromagnetic waves, sound waves within the audible range, and other cases. It has applications in medical imaging, lithotripsy, non-destructive testing, communications as well as many other areas.

\subsection{Basic Theory}

In a bounded domain, $\Omega$, let $h_{A B}(t)$ represent the measured velocity of a material point, $B$, resulting from the application of an impulse of force applied at time $t=0$ at point $A$. The reciprocity principle lets us interchange the sensor and the actuator, so that the signal recorded at $A$ due to an impulse of force applied at $B$ is the same, that is, $h_{A B}(t)=h_{B A}(t)$. Suppose now that the force applied at $B$ is the response at $A$ to an impulse applied at $B$, but inverted and shifted in time by $T$, that is, $f_{B}(t)=h_{A B}(T-t)$, the velocity at a point $\mathrm{C}$ is given by

$$
\begin{aligned}
v_{C}(t) & =f_{B}(t) \otimes h_{B C}(t)=h_{A B}(T-t) \otimes h_{B C}(t) \\
& =\int_{0}^{t} h_{A B}(T-\xi) h_{B C}(t-\xi) \mathrm{d} \xi
\end{aligned}
$$


a measurement b time reversal

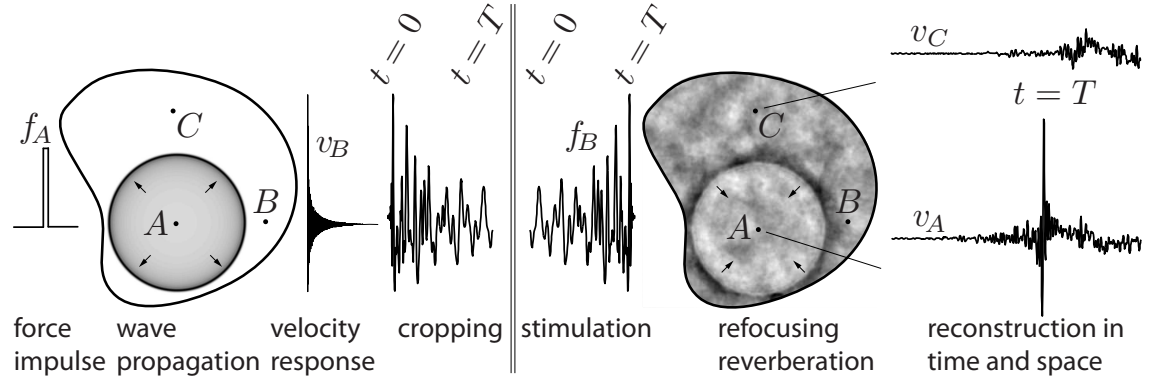

Fig. 1. Time-reversal applied to a reverberant cavity. a) The velocity response at $B$ of a force impulse applied at $A$ is recorded and the initial portion of the signal length $T$ is cropped. b) The signal is time-reversed and used an actuator signal in $B$. Waves propagate and reverberate to eventually refocus in $A$. Perfect reconstruction would entail an infinitely long window and the absence of transducer noise. In practice, the response separates into a signal, as in $A$, and a background noise, as in $A$ and $C$.

where $\otimes$ denotes the convolution operation. If $h_{A B}$ and $h_{B C}$ are not correlated, waves interfere non-constructively, giving a background noise that can be modeled by a random signal with zero-mean velocity and standard deviation $\sigma$ [18]. Applying the reciprocity principle, setting $C=A$, and time $t=T$ in (1) gives,

$$
v_{A}(T)=\int_{0}^{T} h_{A B}^{2}(T-\xi) \mathrm{d} \xi .
$$

The interference is now constructive, yielding a peak of signal localized in space and in time. This process is graphically represented in Fig. 1 for a two dimensional domain.

\section{$3 \quad$ Physical and Engineering Tradeoffs}

A time-reversal set-up typically involves an array of transducers located at the periphery of the domain of interest. The number of transducers can even be reduced to one when the domain has reverberant properties and if the transducer has sufficient bandwidth. The reduction of the number of transducers, which comes with possible gains in implementation complexity, also comes with a cost in performance as illustrated in Fig. 2a. Sets, connected or not, and of any measure, can theoretically be reconstructed. Multi-digit, whole hand stimulation, etc, thus does not differ in principle from the single region example illustrated in Fig. 2b. Repetitions in time are easily achieved by convolution of the signals by a Dirac comb. All these possibilities are crucially dependent on achieving sufficient resolution in time, space, and signal magnitude. It is therefore important to develop, from first principles, the basic tradeoffs involved. 


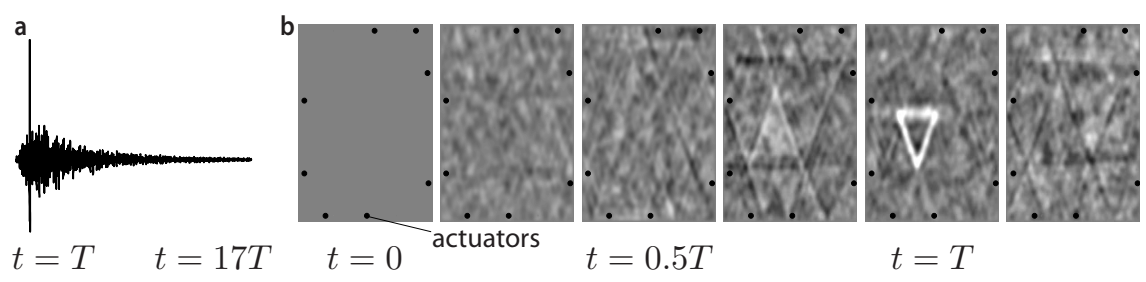

Fig. 2. a) A highly reverberant cavity with a single actuator can yield good reconstruction quality in short time, but with a long tail of noise. b) Example of a triangle reconstructed from eight impulse responses.

\subsection{Contrast Ratio}

The ratio $C=v_{A}(T) / \sigma$ is called contrast, or signal-to-noise ratio, at point $A$. Intuitively, this ratio increases with the introduction of higher modes that have smaller wavelengths. According to the time-frequency uncertainty principle, the time needed to resolve two frequencies $\Delta t$, is inversely proportional to their difference, $\Delta f$, that is, $\Delta t \Delta f \sim 1$. A longer time-reversed window, $T$, makes it possible to resolve mode separated by smaller $\Delta f$ 's, thus increasing contrast. With one actuator in a chaotic cavity [19], or in a scattering medium [18], contrast increases with $\sqrt{T}$. Higher modes become impossible to resolve with the consequence that, when $T$ approaches their decay characteristic time, $\tau$, contrast no longer follows a square root law and reaches a limit. With $N$ actuators, however, simultaneously reproducing inverted impulse responses sum their contributions at any point. Contrast, then, follows the trend $C \propto \sqrt{N T}$, as long as $T<\tau$, which is equivalent to increasing the length of the time-reversed window.

\subsection{Cavity Reverberation}

Cavity having the longest characteristic time possible would seem to be advantageous at first sight. During the reconstruction process, waves converge toward the reconstructed set and then diverge once $t>T$. The time during which diverging waves reverberate before dying out is determined by $\tau$. Repetition of the process at rate faster than $1 / \tau$, will cause noise to accumulate, lowering contrast. The reverberation characteristic time, $\tau$, should therefore be of the same order than $T / N$ to achieve good contrast ratio while raising the reconstruction frequency. A larger number of actuators makes it possible to design the cavity with a shorter reverberation characteristic time.

\subsection{Spatial Resolution}

A factor which must be considered when applying the time-reversal approach to tactile stimulation is that of spatial resolution. It is driven by the smallest reconstructed spot of diameter, $\epsilon$, which is bounded by the smallest wavelength, $\lambda^{-}=c / f^{+}$, where $c$ is the medium's wave propagation celerity, and $f^{+}$the frequency used in the re-focusing process. For a same displacement amplitude, 
smaller wavelengths can be achieved either by increasing the range of frequencies or by lowering propagation celerity. The focused impulse duration, $\zeta$, corresponds to the inverse of the largest frequency used in the re-focusing process giving, $\zeta<\epsilon / c$. A low celerity has an additional benefit. A given amplitude can be achieved with lower frequencies and hence with less power.

\subsection{Medium Deformation}

The focused point size is given by the smallest wavelength used in the reconstruction process and amplitude must be sufficient to fall into the range tactile sensibility. The propagation medium must therefore be able sufficient displacement gradients, that is, deformation. As exemplified in Section 4, the Young's modulus of a material and the smallest achievable bending radius of a membrane are not independent quantities, suggesting that more elastic materials employed in manufacturing of the propagation medium are favorable, lowering propagation velocity, shortening wavelengths, and achieving higher deformations.

\subsection{Power Transmission to a Finger}

Stimulation techniques must transfer power into the load. Here, the stimulated finger perturbs wave propagation when it is coupled to the medium, causing phase shifts and wave front attenuation. To gauge these effects, we developed a simple model of interaction between a finger and flexural waves, see Fig. 3. The finger is lumped into a mass-spring-damper system and waves propagate in an infinite strip of width, $l$, representing the width of the contact. An incident wave of wavelength, $\lambda=2 \pi / k$, amplitude, $A_{I}$, propagates at celerity, $c=\omega / k$, along the $x$-axis, $u_{I}(x, t)=A_{I} e^{j(\omega t-k x)}$. There are two other waves, $u_{R}(x, t)=$ $A_{R} e^{j(\omega t-k x)}$ and $u_{T}(x, t)=A_{T} e^{j(\omega t-k x)}$, the reflected wave and the transmitted wave, respectively. The power transported by $u_{I}$ is $P_{I}=c^{2} k^{2} Z_{\mathrm{S}}\left|A_{I}\right|^{2}$, where $Z_{\mathrm{S}}=f_{\text {act }} / v_{I}$ is impedance of the strip at the point of actuation and $v_{I}=\partial u_{I} / \partial t$ is the out-of-plane velocity [20].

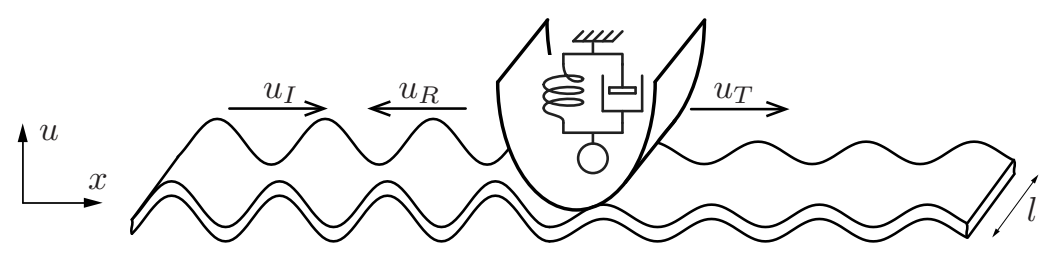

Fig. 3. Model of wave-finger interaction

At $x=0$, finger displacement has the amplitude of the transmitted wave, $u_{\mathrm{F}}(t)=u_{T}(0, t)$. Enforcing continuity of displacement and conservation of momentum at $x=0$ gives,

$$
A_{\mathrm{F}}=A_{T}=A_{I} \frac{2 Z_{\mathrm{S}}}{Z_{\mathrm{F}}+2 Z_{\mathrm{S}}}, \quad A_{R}=-A_{I} \frac{Z_{\mathrm{F}}}{Z_{\mathrm{F}}+2 Z_{\mathrm{S}}},
$$


Table 1. Strip material properties and power needed to displace a fingertip by $10 \mu \mathrm{m}$

\begin{tabular}{lccccc}
\hline Properties & $E$ & $h$ & $\rho_{l}$ & $R_{l}$ & $P$ \\
& $\mathrm{GPa}$ & $\mathrm{mm}$ & $\mathrm{kg} / \mathrm{m}^{3}$ & $\mathrm{~N}$ & $\mathrm{~W}$ \\
\hline Glass & 69.0 & 1.0 & 2300 & 0.0 & 500 \\
BOPET & 4.0 & 0.125 & 1400 & 2.0 & 1.5 \\
\hline
\end{tabular}

where $Z_{\mathrm{S}}$ is the lumped impedance of the strip at the contact and $Z_{\mathrm{F}}=\beta+$ $j[\mu(c k)-\kappa /(c k)]$ is the impedance of the finger where, $\mu, \beta$, and $\kappa$ are mass, damping, and stiffness respectively. Expressing the power of the incident wave as a function of the finger displacement gives,

$$
P_{I}=\frac{c^{2} k^{2}}{4} \frac{\left|Z_{\mathrm{F}}+2 Z_{\mathrm{S}}\right|^{2}}{\left|Z_{\mathrm{S}}\right|}\left|A_{F}\right|^{2},
$$

showing that slow propagation celerity achieves the same displacement for less power. It shows also that $Z_{\mathrm{S}}$ should be larger than $Z_{\mathrm{F}}$ to prevent muffling. On the other hand, as is well known, optimal transmission is when $Z_{\mathrm{F}}=Z_{\mathrm{S}}$. For an isotropic thin strip under uniform traction,

$$
Z_{s}=\sqrt{\rho_{l}\left(R_{l}+D_{l} k^{2}\right)}, \quad \text { and } \quad c=\sqrt{1 / \rho_{l}\left(R_{l}+D_{l} k^{2}\right)}
$$

where the lineic bending stiffness is, $D_{l}=\frac{1}{12} E h^{3} l$, and where, $E$, is the strip Young's Modulus, $l$, the width, $h$, the thickness, $\rho_{l}$, the lineic mass density, and $R_{l}$, the lineic tension. This simple model, while ignoring scattering and finite contact size effects, indicates that if a membrane is used as transmission medium, its material should have high density and low stiffness. Assuming reasonable values for a fingertip impedance, $\mu=0.2 \mathrm{~g}, \beta=1.0 \mathrm{~N} \cdot \mathrm{s} / \mathrm{m}$ and $\kappa=1.0 \mathrm{~N} / \mathrm{mm}$, see $[21], \lambda=15 \mathrm{~mm}, l=10 \mathrm{~mm}$, we can evaluate the appropriateness of some materials for the application of time-reversed acoustics to tactile stimulation in terms of the power needed to displace the skin of a finger, see Table 1.

\section{Preliminary Validation}

A $150 \times 150 \times 0.125 \mathrm{~mm}$ sheet of BoPET (biaxially-oriented polyethylene terephthalate) was stretched and glued to a rigid frame with a surfacic tension of $R_{s}=$ $30 \mathrm{~N} / \mathrm{m}$. For a membrane, $D_{l}$ must be replaced in (5) by $D_{s}=\frac{1}{12} E h^{3} /\left(1-\nu^{2}\right)$, where $\nu$ is the Poisson's ratio of the material. Setting $E=4 \mathrm{GPa}$ and $\nu=0.3$, we obtain a wave celerity varying from 34 to $43 \mathrm{~m} / \mathrm{s}$ for wavelengths varying from $\infty$ to $15 \mathrm{~mm}$, that is frequencies from 0 to $3 \mathrm{kHz}$. The first mode was at $150 \mathrm{~Hz}$ and the characteristic time was about $70 \mathrm{~ms}$. Eight custom-made, miniature, moving-magnet electromagnetic devices $(6.2 \Omega, 1.9 \mathrm{mH}, b l=6 \mathrm{~N} / \mathrm{A}$, $1.5 \times 0.5 \mathrm{~mm}$ ) impinged on the membrane. They were placed in a configuration that avoided symmetries as indicated in Fig 4. The devices were used as force transducers in actuation mode and as velocity transducers in sensing mode. 


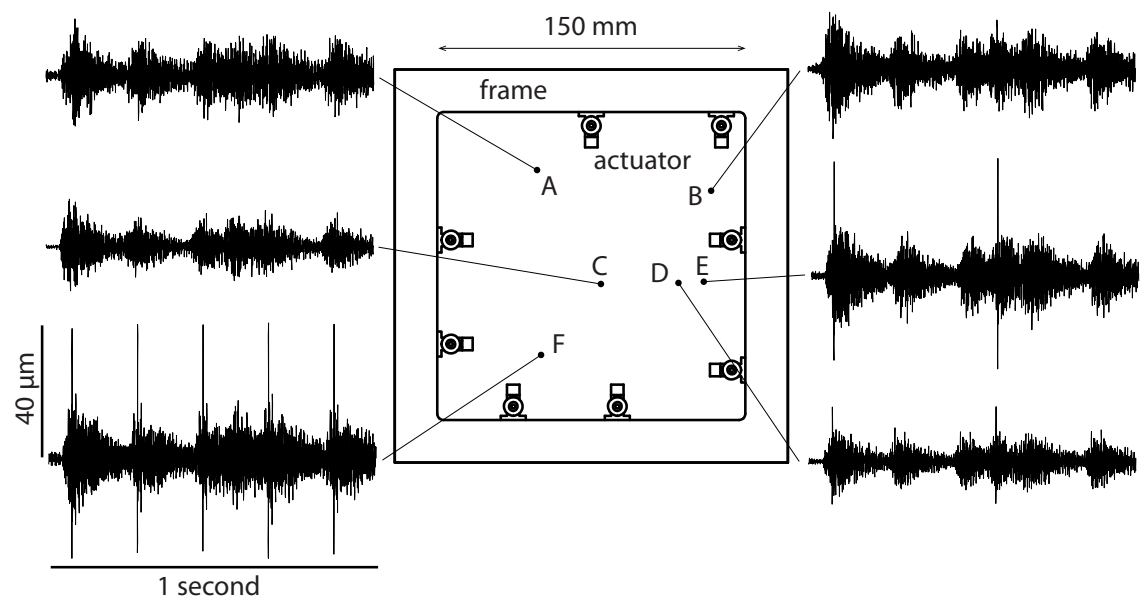

Fig. 4. Experimental set-up and focusing results, at $5 \mathrm{~Hz}$ in $\mathrm{E}$ and at $2 \mathrm{~Hz}$ in $\mathrm{D}$

A two-dimensional cavity of surface area $S$, perimeter $P$ and wave celerity $c(f)$, has $M$ mode below a frequency $f$ according to $M(f)=\pi S(f / c)^{2}+$ $(P f) /(2 c)$, see [22]. It follows that the separation, $\Delta f$, between two modes is given by $2 c^{2} /(4 \pi S f+P c)=2 c^{2} /(4 \pi S c k+P c)=2 c /(2 S k+P)$. Following the discussion of Section 3.1, the time reversal window duration was set at $T \sim\left(2 S k_{\max }+P\right) /\left(2 N c_{\min }\right) \simeq 30 \mathrm{~ms}$.

Waves were re-focused onto two points, $\mathrm{E}$ and $\mathrm{F}$ at 2 and $5 \mathrm{~Hz}$, respectively to re-create time-space impulses. Displacements were recorded by a laser vibrometer and synchronized with the emissions. The results are plotted in Fig 4 for various locations on the membrane. The pulses in $\mathrm{E}$ and $\mathrm{F}$ achieved $40 \mu \mathrm{m}$ in amplitude. At any other point, background noise was present with same amplitude as in $E$ and $\mathrm{F}$.

For the same contrast ratio, the peak amplitude could reach $200 \mu \mathrm{m}$ by scaling up the signal. When a finger touched the surface the amplitude of the signal and of the noise was attenuated by a factor 5 . It was also possible to scale down the signals so that the background noise fell below the tactile sensitivity while keeping the sensation experienced in $\mathrm{E}$ and $\mathrm{F}$ above threshold. The signal bandwidth fell within the audible range and could be heard, but since the sound was a reproduction of an actual impact, it did sound exactly like an impact. The tactile and auditory experiences were therefore mutually coherent.

\section{Conclusion}

Computational time reversal was successfully applied to focusing flexural waves to a spot of similar size to a finger contact, and with amplitudes compatible with the tactile sensitivity range. Further research will aim at increasing the contrast ratio to obtain more vivid stimuli, keeping in mind that the stretched membrane approach is only one among many other options for a propagation medium. 
The ability of reconstructing arbitrary waveforms within arbitrary sets can be approached either by pre-computing response libraries or in a discrete manner or applying the appropriate interpolation laws, or by the development of a model which is sufficiently accurate and computationally acceptable to calculate impulse responses offline, or even online, using advanced computational methods.

\section{References}

1. Gault, R.H.: Tactual interpretation of speech. The Scientific Monthly 22(2), 126-131 (1926)

2. Gault, R.H.: Recent developments in vibro-tactile research. Journal of the Franklin Institute 221, 703-719 (1936)

3. Takasaki, M., Nara, T., Tachi, S., Higuchi, T.: A tactile display using surface acoustic wave with friction control. In: International Workshop on Micro Electro Mechanical Systems, pp. 240-243 (2001)

4. Takasaki, M., Kotani, H., Nara, T., Mizuno, T.: Transparent surface acoustic wave tactile display. In: Proceedings of the IEEE/RSJ International Conference on Intelligent Robots and Systems, pp. 1115-1120 (2005)

5. Watanabe, T., Fukui, S.: A method for controlling tactile sensation of surface roughness using ultrasonic vibration. In: Proceedings of the IEEE International Conference on Robotics and Automation, pp. 1134-1139 (1995)

6. Winfield, L., Glassmire, J., Colgate, J.E., Peshkin, M.: T-PaD: Tactile pattern display through variable friction reduction. In: Proceedings of the Second Joint EuroHaptics Conference and Symposium on Haptic Interfaces for Virtual Environment and Teleoperator Systems, World Haptics 2007, pp. 421-426 (2007)

7. Biet, M., Giraud, F., Lemaire-Semail, B.: Squeeze film effect for the design of an ultrasonic tactile plate. IEEE Transactions on Ultrasonics, Ferroelectrics and Frequency Control 54(12), 2678-2688 (2007)

8. Chubb, E.C., Colgate, J.E., Peshkin, M.A.: Shiverpad: A glass haptic surface that produces shear force on a bare finger. IEEE Transactions on Haptics 3(3), 189-198 (2010)

9. Amberg, M., Giraud, F., Semail, B.: Interface tactile vibrante transparente. French patent 1153963, Laboratoire d'électrotechnique et d'électronique de puissance (L2EP) (L2EP) (May 2011)

10. Strong, M.S., Troxel, D.E.: An electrotactile display. IEEE Transactions on ManMachine Systems 11(1), 72-79 (1970)

11. Bau, O., Poupyrev, I., Israr, A., Harrison, C.: TeslaTouch: electrovibration for touch surfaces. In: Proceedings of the 23nd Annual ACM Symposium on User Interface Software and Technology, pp. 283-292 (2010)

12. Tang, H., Beebe, D.J.: A microfabricated electrostatic haptic display for persons with visual impairments. IEEE Transactions on Rehabilitation Engineering 6(3), 241-248 (1998)

13. Iwamoto, T., Akaho, D., Shinoda, H.: High resolution tactile display using acoustic radiation pressure. In: Proceedings of SICE Annual Conference, pp. 1239-1244 (August 2004)

14. Hoshi, T., Iwamoto, T., Shinoda, H.: Non-contact tactile sensation synthesized by ultrasound transducers. In: Proceedings of the World Haptics Conference, pp. 256-260 (2009) 
15. Fink, M.: Time reversed acoustics. Physics Today 50, 34 (1997)

16. Montaldo, G., Tanter, M., Fink, M.: Real time inverse filter focusing through iterative time reversal. Journal of the Acoustical Society of America 115(2), 768-775 (2004)

17. Fink, M.: Time reversal of ultrasonic fields. i. basic principles. IEEE Transactions on Ultrasonics, Ferroelectrics and Frequency Control 39(5), 555-566 (1992)

18. Derode, A., Tourin, A., Fink, M.: Limits of time-reversal focusing through multiple scattering: Long-range correlation. The Journal of the Acoustical Society of America 107, 2987 (2000)

19. Quieffin, N., Catheline, S., Ing, R.K., Fink, M.: Real-time focusing using an ultrasonic one channel time-reversal mirror coupled to a solid cavity. The Journal of the Acoustical Society of America 115, 1955 (2004)

20. Morse, P.M.C., Ingard, K.U.: Theoretical acoustics. Princeton University Press (1986)

21. Wiertlewski, M., Hayward, V.: Transducer for mechanical impedance testing over a wide frequency range through active feedback. Review of Scientific Instruments (in press, 2012)

22. Blevins, R.D.: Modal density of rectangular volumes, areas, and lines. The Journal of the Acoustical Society of America 119(2), 788 (2006) 